\title{
The human dimension: putting the person into personalised medicine
}

\author{
Rob Horne, Professor of Behavioural Medicine, UCL, CASMI
}

\begin{abstract}
Technological advances enabling us to personalise medical interventions at the biological level must be matched by parallel advances in how we support the informed choices essential to patient and public participation. We cannot take participation for granted. To be truly personalised, medicine must take account of the perceptions and capabilities that shape participation. To do this, we need a better understanding of how people perceive personalised medicine and how they judge its value and risks. To realise the promise of personalised medicine $4 \mathrm{P}$ medicine we need to personalise at the psychosocial as well as biological dimension, putting the person personalised medicine.
\end{abstract}

KEYWORDS. Personalised medicine, stratified medicine, 4P medicine, patient and public perceptions, idiosyncrasia, beliefs, psychosocial aspects, Necessity Concerns Framework, illness perceptions, engagement, behaviour

\section{Introduction}

The idea of personalising treatment is at the core of medicine and can be traced back to the teachings of Hippocrates(Sykiotis et al., 2005). Hippocrates' therapeutic strategy was based on understanding the idiosyncrasia - the unique characteristics of the individual determining response to the disease and treatment. Identifying the patient's idiosyncrasia enables tailored treatment by 'making changes in drugging or in regiment to suit the several conditions of age, season, physique and disease'(Jones, 1931, Sykiotis et al., 2005). In recent years, the concept of personalised (or stratified) medicine has been reinvigorated by scientific advances in genomics promising treatment approaches tailored to the genetic characteristics of the individual(Horne et al., In Preparation).

Taken together, advances in genomics, data-analytics and e-health technologies offer the prospect of 4P Medicine that is predictive, pre-emptive, personalised and participatory(Horne and Hankins, In Submission). We are now able to characterise Hippocrates' idiosyncrasia more accurately than ever before. With this, 4P medicine promises to take us beyond the one size fits all, population-based strategy that has produced the 'blockbuster drugs' of the past, to tackle the rising tide of chronic disease in ageing populations and re-orientate health services from a focus on treating established diseases (often when it is too late) to the maintenance of health. 


\section{The ghost in the machine: extending personalisation to include psychosocial aspects of the person}

Participation is at the centre of the 4P model, and rightly so (see Figure 1). But how will this work in practice?

Insert fig 1 here

It is often assumed that widespread access to medical data, diagnostic tests and health maintenance apps, coupled with the democratization of medical knowledge, will empower individuals to self-manage health and illness. However, this level of participation is unlikely to occur if left to chance on the assumption that it will emerge as a corollary of the scientific evidence supporting biomedical personalization (Horne et al., 2015).

To make 4P work we need to extend our focus beyond the biological and technological to take account of the psychosocial factors that make us unique and influence health behaviours and outcomes. In recognition of this principle, Gorini and Pravettoni have called for the addition of a fifth ' $P$ ' (psycho-

Box 1 - Characterisation of 4P medicine

Winston Orwell has just returned from his daily $10 \mathrm{Km}$ run. Scrutinizing his svelte $70 \mathrm{Kg}$ form in front of his interactive bathroom mirror, he asks BioSys (affectionately known as Big Sister) to activate his health check screen. The info graphic shows that his health profile indices are all in the green zone. Prometheus, his medication monitor, reminds him that his daily dose of Tailoran is due. He pops the pill and reflects on taking Tailoran for 40 years since it was prescribed in response to his genetic risk, tailored to his pharmacodynamic and pharmacokinetic profile. Winston is 98 years old.

cognitive) to the 4P model to increase its feasibility(Gorini et al., 2008). To be truly personalised (and hence, more effective) treatment must address two aspect of personalisation: biological and psychosocial.

\section{Potential disconnects between the person and personalised medicine}

Consider an imagined outcome of 4P shown in Box 1. Although exaggerated, this characterisation provides a frame for discussing some of the psychosocial challenges to 4P medicine and how these might be addressed by connecting biomedical and psychosocial aspects of personalisation. The key issue is the encounter of the individual with the specific healthcare service or treatment. In Winston's case, we are looking at the interaction of the person with personalised medicine. 
When healthcare is made available to the individual, engagement is essentially determined by a, sometimes complex, interplay between motivation and ability. There are a number of potential disconnects between the person and personalised medicine which might reduce motivation and/or ability to participate in $4 \mathrm{P}$ medicine.

\section{Motivation to participate: the importance of public perceptions of value and risk}

We see from Winston's morning encounter with Bio Sys an active routine engagement with health maintenance requiring daily monitoring, exercise and taking Tailoran, a preventative medication. The scenario implies that they have become a regular healthy habit, so we assume that Winston's economic circumstances allow the time and space to perform these tasks. This assumption highlights a challenge to realising the promise of $4 \mathrm{P}$ medicine: the question of equality of access. A full discussion is beyond the scope of this paper though the issue is dealt with in more detail elsewhere(Barker, 2016). However, if we think back to the personal level and the question of participation, we see that although Winston's health maintenance behaviours have become routine, establishing the routine would have required motivation. Can we assume that the motivation to participate in the predictive and pre-emptive health maintenance envisioned by 4P medicine will be an automatic corollary of the technological capability? Based on the evidence for public engagement in current preventative health measures the answer is 'probably not'.

As with most innovations there will be early enthusiastic adopters but others may need more convincing. What might this entail? Simply presenting Winston with the evidence for the benefits of prevention and the risks of doing nothing may not be enough. The idea that health-related behaviour arises from rational choices based on objective assessments of the evidence is challenged by research in behavioural economics that choices are influenced by an array of psychological and environmental factors, including systematic biases in how we interpret and value information or evidence (Kahneman, 2011, Tversky and Kahneman, 1985).

Technological advances enabling us to personalise medical interventions at the biological level must be matched by parallel advances in how we support the informed choices essential to patient and public participation. We cannot take participation for granted. To be truly personalised, medicine must take account of the perceptions and capabilities that shape participation. To do this, we need a better understanding of how people perceive personalised medicine and how they judge its value and risks.

\section{Prevention is better than cure but less motivating}

For many health as value is defined more by its absence than presence(Lau et al., 1986). For many, when they feel well, matters of health and illness tend to recede into the background. Our attention is focused on health more when we become ill or when we fear losing it. This may be a poor spring board for participation in $4 \mathrm{P}$ medicine where targeted pre-emption is predicated on early action that is presumably sustained over the long-term.

The ability to predict future disease is core to 4P medicine and is becoming a reality. But little is known about whether people will want this information or how it might influence behaviour and well-being. Do people want to know their genetic risk and will knowledge of risk be sufficient to motivate the 
behaviours necessary to mitigate the risk? Effects of risk information on behaviour may be paradoxical and influenced by erroneous beliefs about disease and treatment. There is a common perception that genetic risk is immutable and this might weaken beliefs in the efficacy of intervention and reduce the motivation to adopt preventative behaviours(Marteau and Lerman, 2001). However, little is known about public perceptions of the genetic risk information that underpins pre-emption in 4P medicine or the public's willingness to engage in the privative necessary to avoid them.

\section{Nonadherence to treatment: the importance of common-sense evaluations of illness and treatment}

The World Health Organisation has estimated that about half of medicines prescribed for long term conditions are not taken as advised(Sabaté, 2003). There are many, complex reasons for this but as with most other behaviours adherence is a product of motivation, capability and opportunity. To understand why a person might follow a treatment recommendation over the long-term, we need to understand the perceptions that underpin motivation as well as the practicalities that determine access and ability to adhere. There is, of course, a degree of overlap between these factors: we may be more motivated to do something that is easy and accessible being motivation might help us to overcome certain barrier .s.However, studies of adherence have consistently shown that nonadherence is often related to peoples' personal beliefs about illness and treatment, which may be at odds with the medical view or scientific evidence(Horne et al., 2005).

A recent systematic review and meta-analysis showed that providing information about the benefits and harms of medication had no overall impact on decisions about whether to start and continue medicines, although it increased knowledge and make people feel more comfortable about their decision(Crockett et al., 2011). One reason for this finding is that decisions about medicines are not influenced by the scientific evidence alone but also by the beliefs and values that the person brings to the encounter with the prescription(Horne et al., 1999, Horne and Weinman, 1999).

\section{Making decisions about taking medicines: The Necessity Concerns Framework}

Studies of adherence to prescribed medicines consistently link that nonadherence to patients' beliefs andin particular, the way in which the individual judges their personal need for the medicine (necessity beliefs) relative to their concerns about taking it.(Horne et al., 2013)

Necessity beliefs might be thought of as the answer to two questions: 'How much do I need this treatment to achieve a goal that's important to me?' and 'How much can I get away without it?'. We ask the latter question because pharmaceuticals are commonly perceived as a 'double-edged sword' where therapeutic efficacy goes hand in hand with the potential for harm(Horne et al., 1999, Horne, 1999). This representation might be traced back to the Ancient Greeks who had one word for medicine and poison: pharmakon, the origin of 'pharmaceutical'. It may also stem from a more basic aversion to taking regular medication(Pound et al., 2005), or even to the meaning we attach to taking regular treatments and its impact on our sense of self or how we think others see us(Cooper et al., 2002). Perceived necessity is not a form of efficacy belief: we might believe that a treatment will be effective but not that we need it. We might have a low necessity belief even if we understand the scientific evidence for the potential 
benefits of treatment. This might occur because we do not 'value' that particular benefit or perceive it be important enough to overcome our concerns about taking the medicine.

We might question how Winston is convinced of his personal need to take Tailoran for 40 years. Most people do not blindly follow treatment advice. Rather, we evaluate the value of the advice and make a judgement on whether we need to follow it. This applies even when we trust the doctor. A nonadherent patient might trust the doctor but not the prescription. Evaluations of personal need for the treatment are influenced by common-sense understanding of the condition for which the treatment is prescribed. To be convinced of a personal need for ongoing medication, we must first perceive a good fit between our conception of the problem (the illness or condition) and the solution (the medicine) (Horne and Weinman, 2002). Here, symptom perceptions relative to expectations are key(Horne and Weinman, 2002). The treatment proposition may be more convincing when we experience symptoms that we perceive to be relieved by the medicine than if we are taking the medicine to prevent a condition from arising(Cooper et al., 2009).

Before we are diagnosed with a chronic illness, most of our experience of illness is symptomatic and acute. However, for many long-term conditions, and presumably many of the pre-emptive treatments in 4P medicine, the medical rationale for treatment is based on a prophylaxis model where the benefits of treatment are often 'silent' and realised in the long-term. This may be in stark contrast to our intuitive model of 'no symptoms; no problem'(Halm et al., 2006). Similarly, missing doses may not lead to an immediate deterioration in symptoms, reinforcing the erroneous perception that high adherence to the medication may not be necessary. Related to this is the fact that people often stop taking treatment when they judge that the condition has improved, usually if symptoms ameliorate.

Information about the potential benefits of treatment may be less persuasive than 'concrete' symptom experiences. This is illustrated by a study exploring the reasons why people decided not to take a clinically indicated offer of antiretroviral treatment (ART) for HIV infection. Evidence-based guidelines for the optimum time to initiate ART stipulate CD4 count (an indicator of immune status) and viral load (a marker for disease activity) as key indicators for when ART is clinically indicated. However, receiving 'abstract' information about personal CD4 and viral load lab results was less persuasive than more 'concrete' symptom experiences. A common reason given by interviewees for refusing ART was that they were experiencing few, if any, of the symptoms that they associated with HIV-infection. Their common-sense interpretation of their experiences (feeling fine) seemed to convince them that they did not need treatment yet and could afford to delay starting ART, despite the scientific evidence supporting an immediate start.(Horne et al., 2007)

Concerns about medication There is a striking similarity in the type of concerns that patients report about prescription medicines. One obvious source of concern is the experience of symptoms as medication 'side-effects' and the disruptive effects of medication on daily living; but this is not the whole picture (Cooper et al., 2015). Many patients receiving regular medication who have not experienced adverse effects are still worried about possible problems in the future. These concerns often arise from the belief that regular use can lead to dependence or that the medication will accumulate within the body and lead to long-term effects(Horne and Weinman, 2002). Concerns also relate to the meaning that being on regular medication has for the individual and their sense of self(Cooper et al., 2002). Concerns about specific medicines are also related to more general beliefs about pharmaceuticals as a class of treatment. Many people seem to have a fairly negative orientation 
to pharmaceuticals, perceiving them to be fundamentally harmful, addictive substance that should not be taken for long periods of time but that tend to be over-prescribed by doctors(Horne et al., 1999). Moreover, the dangerous aspects of medication are often linked to their chemical/unnatural origins and to suspicions of the pharmaceutical industry and scientific medicine (Horne et al., 1999) (Britten et al., 2010, Pound et al., 2005). In experimental studies, people with more negative views about pharmaceuticals in general are more likely to think that symptoms are caused by a specific drug (attribute symptoms as side-effects)(Heller et al., 2015) and less likely to recall side effects correctly.(Heller et al., in press)

\section{Public perceptions of personalised medicine}

The adherence literature points to the existence of a profound disconnect between a deterministic view of treatment based on scientific medicine and the 'common-sense' evaluations of illness and treatment of many patients. But are these issues likely to be relevant for personalised medicine which, promises to be more effective and less harmful; because it is targeted to the biological characteristics of the individual? To answer this question, we need more research into patient and public perceptions and behaviour in relation to personalised medicine. But the early indicators are that biological personalisation may not guarantee behavioural participation.

Consider two illustrative examples. First, the anticancer drug Imatinib is an example of a personalised medicine, prescribed for patients with Philadelphia chromosome positive chronic myeloid leukaemia(Issa, 2007). In a study of adherence to Imatinib, Marin and colleagues found that $26 \%$ of patients were nonadherent with nonadherence as the only predictor of poor clinical response to the drug(Marin et al., 2010). This study did not assess the reasons for nonadherence but in a separate interview-based study patients nonadherence to Imatinib was associated with patients beliefs(Eliasson et al., 2011). A second indicator that participation may not be an automatic corollary of personalised medicine was provided by an analogue study of public attitudes to hypothetical treatments for glandular fever. A medicine personalised to the genetic profile of the individual was perceived as no more effective and no less harmful than a pharmaceutical medicine and significantly less harmful than a natural remedy(Green et al., 2013).

\section{The problem of the 'B' Patient}

A further issue arises in relation to 'molecularly unstratified' or ' $\mathrm{B}$ ' patients. Personalised medicine (PM) involves tailoring medical treatment based on the individual's biological data. Implicit in the notion of targeting a treatment to a particular genetic profile is that the process of tailoring will identify patients who do not have the genetic profile that fits the drug: a ' $\mathrm{B}$ ' Patient. When the drug offers the potential to stay the course of an otherwise fatal condition, how we do we ameliorate the consequences of informing a ' $\mathrm{B}$ ' patientthat they are not eligible for the treatment? In parallel with the development of technological aspects of biological personalisation, we require equally innovative approaches to the management of patients who are not eligible for genetically targeted treatments, including how we might ameliorate the psychological effects of being in the B stream for life-saving treatments. The need for humane end of life care that is tuned to psychological and spiritual needs will remain even in the era of $4 \mathrm{P}$ medicine (see Hordern this volume for a more detailed discussion). 


\title{
Conclusions
}

In conclusion, biomedical advances, although fundamental, cannot deliver on the promise of personalised medicine in isolation. We now have the capacity to characterise Hippocates idiosyncrasia more accurately from the biological perspective, than ever before. But the promise of personalised medicine will only be fully realised if we develop better methods for characterising the psychosocial idiosyncrasia influencing engagement and outcome. Good prescribing is about the application of psychology as well as pharmacology.

We should tailor the prescription to take account of the patients' beliefs and preferences. This is essential if we are to support informed treatment choices. Informing should be an active process, which involves more than simply presenting the evidence. It also entails eliciting the patient's beliefs and identifying whether pre-existing beliefs might act as a barrier to an unbiased interpretation of the evidence. If the interpretation of information is influenced by misconceptions about the illness and treatment, then can the choice be truly informed?(Horne, 2006) Addressing psychosocial aspects of the patients encounter with the treatment might also have direct effects of outcome by enhancing the nonspecific beneficial effects ('placebo' component) and reducing the non-specific harmful effects ('nocebo' component) of pharmacologically active treatments(Rief et al., 2011). Although these approaches are in their infancy, personalising information about medicines to take account of individual patients' doubts and concerns can be achieved in brief and cost-efficient interventions that improve engagement with essential treatments (Clifford et al., 2006, Elliott et al., 2008, Petrie et al., 2012). To realise the promise of personalised medicine $4 \mathrm{P}$ medicine we need to personalise at the psychosocial as well as biological dimension, putting the person personalised medicine.

WORD COUNT 3,168

\section{ACKNOWLEDGEMENTS}

Sections of this paper were originally prepared as a personal view invited by the Academy of Medical Sciences

KAREN - Add CLAHRC NT statement

\author{
RH Biography
}


DRAFT

Horne-R_The human dimension_.docx 


\section{REFERENCES}

BARKER, R. 2016. Bioscience-Lost in Translation?: How precision medicine closes the innovation gap, Oxford University Press.

BRITTEN, N., RILEY, R. \& MORGAN, M. 2010. Resisting psychotropic medicines: a synthesis of qualitative studies of medicine-taking. Advances in Psychiatric Treatment, 16, 207-218.

CLIFFORD, S., BARBER, N., ELLIOTT, R., HARTLEY, E. \& HORNE, R. 2006. Patient-centred advice is effective in improving adherence to medicines. Pharmacy World and Science, 28, 165-170.

COOPER, V., BUICK, D., HORNE, R., LAMBERT, N., GELLAITRY, G., LEAKE, H. \& FISHER, M. 2002. Perceptions of HAART among gay men who declined a treatment offer: preliminary results from an interview-based study. AIDS Care, 14, 319-28.

COOPER, V., GELLAITRY, G., HANKINS, M., FISHER, M. \& HORNE, R. 2009. The influence of symptom experiences and attributions on adherence to highly active anti-retroviral therapy (HAART): a six-month prospective, follow-up study. AIDS Care, 21, 520-8.

COOPER, V., METCALF, L., VERSNEL, J., UPTON, J., WALKER, S. \& HORNE, R. 2015. Patient-reported side effects, concerns and adherence to corticosteroid treatment for asthma, and comparison with physician estimates of side-effect prevalence: a UK-wide, cross-sectional study. NPJ Prim Care Respir Med, 25, 15026.

CROCKETT, R. A., SUTTON, S., WALTER, F. M., CLINCH, M., MARTEAU, T. M. \& BENSON, J. 2011. Impact on Decisions to Start or Continue Medicines of Providing Information to Patients about Possible Benefits and/or Harms: A Systematic Review and Meta-Analysis. Medical Decision Making, 31, 767-777.

ELIASSON, L., CLIFFORD, S., BARBER, N. \& MARIN, D. 2011. Exploring chronic myeloid leukemia patients' reasons for not adhering to the oral anticancer drug imatinib as prescribed. Leukemia research, 35, 626-630.

ELLIOTT, R. A., BARBER, N., CLIFFORD, S., HORNE, R. \& HARTLEY, E. 2008. The cost effectiveness of a telephone-based pharmacy advisory service to improve adherence to newly prescribed medicines. Pharmacy World \& Science, 30, 17-23.

GORINI, A., GAGGIOLI, A., VIGNA, C. \& RIVA, G. 2008. A second life for eHealth: Prospects for the use of 3-D virtual worlds in clinical psychology. Journal of Medical Internet Research, 10(3).

GREEN, D. W., HORNE, R. \& SHEPHARD, E. A. 2013. Public perceptions of the risks, benefits and use of natural remedies, pharmaceutical medicines and personalised medicines. Complementary Therapies in Medicine, 21, 487-491.

HALM, E. A., MORA, P. \& LEVENTHAL, H. 2006. No symptoms, no asthma: the acute episodic disease belief is associated with poor self-management among inner-city adults with persistent asthma. Chest, 129, 573-80.

HELLER, M. K., CHAPMAN, S. \& HORNE, R. in press. No Blank Slates: Pre-existing Schemas about Pharmaceuticals Predict Memory for Side Effects. Psychology \& Health.

HELLER, M. K., CHAPMAN, S. C. \& HORNE, R. 2015. Beliefs about medication predict the misattribution of a common symptom as a medication side effect - Evidence from an analogue online study. $J$ Psychosom Res, 79, 519-29.

HORNE, R. 1999. Patients' beliefs about treatment: the hidden determinant of treatment outcome? Journal of Psychosomatic Research, 47, 491-5.

HORNE, R. 2006. Beliefs and adherence to treatment: the challenge for research and clinical practice. In: HALLIGAN, P. W. \& AYLWARD, M. (eds.) The Power of Belief: Psychosocial Influence on IIIness, Disability and Medicine. Oxford: Oxford University Press.

HORNE, R., BELL, J. I., MONTGOMERY, J. R., RAVN, M. O. \& TOOKE, J. E. 2015. A new social contract for medical innovation. Lancet, 385, 1153-4. 
HORNE, R., CHAPMAN, S. C. E., PARHAM, R., FREEMANTLE, N., FORBES, A. \& COOPER, V. 2013. Understanding Patients' Adherence-Related Beliefs about Medicines Prescribed for Long-Term Conditions: A Meta-Analytic Review of the Necessity-Concerns Framework. PLoS ONE, 8, e80633.

HORNE, R., COOPER, V., GELLAITRY, G., DATE, H. L. \& FISHER, M. 2007. Patients' perceptions of highly active antiretroviral therapy in relation to treatment uptake and adherence: the utility of the necessity-concerns framework. JAIDS, 45, 334-41.

HORNE, R., DIEPPE, P. \& ARMITAGE, S. In Preparation. The other man's poison: tribal differences in allopathic and complementary practitioners' views about the risks and benefits of pharmacological, manipulative and complementary therapies. .

HORNE, R. \& HANKINS, M. In Submission. The Medication Adherence Report Scale (MARS). Quality \& Safety in Health Care.

HORNE, R. \& WEINMAN, J. 1999. Patients' beliefs about prescribed medicines and their role in adherence to treatment in chronic physical illness. Journal of Psychosomatic Research, 47, 55567.

HORNE, R. \& WEINMAN, J. 2002. Self-regulation and self-management in asthma: exploring the role of illness perceptions and treatment beliefs in explaining non-adherence to preventer medication. Psychology \& Health, 17, 17 - 32.

HORNE, R., WEINMAN, J., BARBER, N., ELLIOTT, R. A. \& MORGAN, M. 2005. Concordance, Adherence and Compliance in Medicine Taking: A conceptual map and research priorities, London, National Institute for Health Research (NIHR) Service Delivery and Organisation (SDO) Programme. http://www.sdo.nihr.ac.uk/sdo762004.html.

HORNE, R., WEINMAN, J. \& HANKINS, M. 1999. The Beliefs about Medicines Questionnaire: the development and evaluation of a new method for assessing the cognitive representation of medication. Psychology \& Health, 14, 1-24.

ISSA, A. M. 2007. Personalized medicine and the practice of medicine in the 21st century. McGill Journal of Medicine, 10, 53-57.

JONES, W. 1931. Hippocrates, Nature of Man. Loeb Classical Library, Volume?]?]? Harvard University Press, Cambridge, Massachusetts.

KAHNEMAN, D. 2011. Thinking, fast and slow. 1st. New York: Farrar, Straus and Giroux.

LAU, R. R., HARTMAN, K. A. \& WARE, J. E. 1986. Health as a value: methodological and theoretical considerations. Health Psychology, 5, 25-43.

MARIN, D., BAZEOS, A., MAHON, F.-X., ELIASSON, L., MILOJKOVIC, D., BUA, M., APPERLEY, J. F., SZYDLO, R., DESAI, R., KOZLOWSKI, K., PALIOMPEIS, C., LATHAM, V., FORONI, L., MOLIMARD, M., REID, A., REZVANI, K., LAVALLADE, H. D., GUALLAR, C., GOLDMAN, J. \& KHORASHAD, J. S. 2010.

Adherence Is the Critical Factor for Achieving Molecular Responses in Patients With Chronic Myeloid Leukemia Who Achieve Complete Cytogenetic Responses on Imatinib. Journal of Clinical Oncology, 28, 2381-2388.

MARTEAU, T. M. \& LERMAN, C. 2001. Genetic risk and behavioural change. BMJ: British Medical Journal, 322, 1056.

PETRIE, K. J., PERRY, K., BROADBENT, E. \& WEINMAN, J. 2012. A text message programme designed to modify patients' illness and treatment beliefs improves self-reported adherence to asthma preventer medication. British journal of health psychology, 17, 74-84.

POUND, P., BRITTEN, N., MORGAN, M., YARDLEY, L., POPE, C., DAKER-WHITE, G. \& CAMPBELL, R. 2005. Resisting medicines: A synthesis of qualitative studies of medicine taking. Social Science \& Medicine, 61, 133-155.

RIEF, W., BINGEL, U., SCHEDLOWSKI, M. \& ENCK, P. 2011. Mechanisms involved in placebo and nocebo responses and implications for drug trials. Clinical Pharmacology \& Therapeutics, 90, 722-726. 
SABATÉ, E. 2003. Adherence to long-term therapies: evidence for action, World Health Organization. SYKIOTIS, G. P., KALLIOLIAS, G. D. \& PAPAVASSILIOU, A. G. 2005. Pharmacogenetic principles in the Hippocratic writings. The Journal of Clinical Pharmacology, 45, 1218-1220.

TVERSKY, A. \& KAHNEMAN, D. 1985. The framing of decisions and the psychology of choice. Environmental Impact Assessment, Technology Assessment, and Risk Analysis. Springer. 